\title{
Un programa de lectura y escritura universitario: Lineamientos teóricos, características y resultados de aplicación'
}

Estela Inés Moyano²
Jacqueline Giudice

Universidad de Flores ${ }^{4}$

Artículo de Investigación

Recibido: Febrero 15 de 2016 Aprobado: Mayo II de 2016

\section{Resumen}

Este artículo tiene por objetivo presentar el Programa de Lectura y Escritura Académicas (PROLEA-UFLO) que se está implementando en la Universidad de Flores (Buenos Aires, Argentina). Para ello, se presentan los lineamientos teóricos que orientan el programa, su metodología de aplicación didáctica, sus características y algunos resultados obtenidos en una instancia de aplicación. Así, se desarrollan primero las razones por las que un programa de este tipo debe ser instalado; una teoría de aprendizaje mediada por el lenguaje; una teoría de género y discurso enmarcada en la Lingüística Sistémico-Funcional que se propone como base para la enseñanza y, como metodología, un dispositivo didáctico adaptado

Este artículo presenta información producida en el marco del proyecto de investigación "Seguimiento y evaluación del impacto de un programa de lectura y escritura académica a lo largo de una carrera universitaria", dirigido por Estela I. Moyano y financiado por la Universidad de Flores. Investigadores: Jacqueline Giudice y Marcelo Godoy. Los objetivos de la investigación son monitorear el PROLEA-UFLO en tres aspectos: evolución de los estudiantes en sus habilidades de lectura y escritura, evolución del dispositivo negociación entre pares y modificaciones en la gestión de las carreras para sostener el Programa.

2 Coordinadora del Programa de Lectura y Escritura Académicas de la Universidad de Flores (PROLEA-UFLO). Correo electrónico: estela.moyano@uflo.edu.ar

3 Profesora del Programa de Lectura y Escritura Académicas de la Universidad de Flores (PROLEA-UFLO). Correo electrónico: jgiudice@uflo.edu.ar

4 Rivadavia 574I, Ciudad Autónoma de Buenos Aires, Argentina. Teléfono: (54 I I) 4433-6300. 
del de la Escuela de Sydney. En segundo término, se presenta la intervención del Programa llevada a cabo en una materia ubicada en el primer cuatrimestre del primer año de la carrera de Psicología, a fin de mostrar la evolución de la escritura de los estudiantes en ese contexto.

Palabras clave: programa de lectura y escritura académicas - Lingüística Sistémico-Funcional - aprendizaje - enseñanza - géneros discursivos - evolución de habilidades de lectura y escritura - universidad.

\title{
A tertiary reading and writing program: theoretical guidelines, characteristics and application results
}

\begin{abstract}
The goal of this article is to present the Academic Reading and Writing Program (PROLEA-UFLO) which is being implemented at Flores University (Buenos Aires, Argentina). For that purpose, the theoretical guidelines that found the program are presented, as well as its didactic application methodology, its characteristics and some of the results achieved in one instance of application. Thus, in the first place the rationale for the establishment of such type of program is developed; a theory of learning mediated by language; a theory of genre and discourse framed within Systemic-Functional Linguistics which is proposed as a basis for teaching and, a didactic device adapted from the Sydney School as the methodology. Secondly, an intervention carried out in the first term of the year in the major of Psychology is exposed in order to show the evolution of the students' written production within that context.
\end{abstract}

Key words: academic reading and writing program, Systemic-Functional linguistics, learning, teaching, discourse genres, reading and writing skills evolution.

\section{Um programa de leitura e escrita universitário. Lineamentos teóricos, características e resultados de aplicação}

\section{Resumo}

Este artigo tem por objetivo apresentar o Programa de Leitura e Escrita Acadêmicas (PROLEA-UFLO) que está sendo implementado na Universidade de Flores (Buenos Aires, Argentina). Para isso se apresentam os lineamentos teóricos que orientam o programa, sua metodologia de aplicação didática, suas características e alguns resultados obtidos numa instância de aplicação. Assim sendo, desenvolvem-se primeiro as razões pelas que um programa de este tipo deve ser instalado; uma teoria da aprendizagem mediada pela linguagem; uma teoria de gênero e discurso emoldurada na Linguística Sistêmico-Funcional que é proposta como base para o ensino e, como metodologia, um dispositivo didático adatado do da Escola de Sydney. Em segundo termo, se apresenta a intervenção do programa levada a cabo numa disciplina programada dentro do primeiro quadrimestre do primeiro ano do curso de Psicologia, com a finalidade de mostrar a evolução dos estudantes neste contexto.

Palavras chave: programa de leitura e escrita acadêmicas, linguística sistêmico-funcional, aprendizagem, ensino, gêneros discursivos, evolução de habilidades de escrita e leitura, universidade. 


\section{Introducción}

Los propósitos de este trabajo son presentar, en primer lugar, los lineamientos teóricos básicos y principales características metodológicas del Programa de Lectura y Escritura Académicas (PROLEA) que se encuentra en la fase de implementación a lo largo de las carreras que se dictan en la Universidad de Flores (UFLO) y, en segundo lugar, un ejemplo de los resultados de su aplicación. Este Programa tiene, como objetivo principal, favorecer el desarrollo de los estudiantes en esta área a lo largo de su carrera universitaria, con el fin de mejorar también el aprendizaje de contenidos en las materias específicas. De este modo, el Programa se propone, en articulación con otros dispositivos que también se están desarrollando en la Universidad, favorecer la inserción, la permanencia y la graduación de los estudiantes de las diferentes carreras.

El trabajo llevado a cabo se entiende como Programa pues incluye actividades de enseñanza de la lectura y la escritura académicas y profesionales a los estudiantes de diferentes carreras dictadas en la Universidad, actividades de gestión que permiten su implementación a lo largo de la currícula y trabajo de investigación que monitorea el avance y los resultados producidos.

A continuación se enunciarán, en primer lugar, una fundamentación para la instalación del Programa, seguida, en segundo término, de los principios y los criterios teóricos que lo fundamentan, tomando especialmente las teorías de género y de discurso propuestas en la Lingüística Sistémico-Funcional así como también los desarrollos pedagógicos elaborados en ese marco, basados en una concepción de aprendizaje mediado por el desarrollo del lenguaje y en supuestos de teorías sociológicas y psicológicas. En tercer lugar, se describirán las características principales del Programa, señalando las ventajas que presenta frente a otras modalidades, como los talleres de escritura paralelos al cursado de una carrera y los talleres a cargo de profesores de las disciplinas específicas. En último término, se dará cuenta de una aplicación en una materia del primer cuatrimestre del primer año de una de las carreras de la Universidad, a fin de mostrar los primeros pasos de evolución de los estudiantes.

\section{Fundamentos para la creación de un Programa de Lectura y Escritura en la Universidad}

En las últimas décadas, se ha generalizado el acuerdo acerca de la necesidad de creación de programas de lectura y escritura académicas que favorezcan el desarrollo de estas habilidades ya no solo en lengua extranjera ${ }^{5}$ sino también en lengua materna ${ }^{6}$. En este sentido, se ha instalado cada vez con mayor fuerza

5 Hyon, S. "Genre in three traditions: implications for ESL". TESOL Quarterly 30(4), 1996, págs. 693-722; Hyland, K. \& Hamp Lyons, L. "EAP: issues and directions". Journal of English for Academic Purposes, I, 2002, págs. I - I2; Bawarshi, A.S. \& Reiff, M.J. Genre. An Introduction to History, Theory, Research and Pedagogy. Colorado/Indiana: Parlor Press and WAC Clearinghouse, 20 I0; Coffin, C. \& Donohue, J.P. (Eds) English for Academic Purposes: contributions from Systemic Functional Linguistics and Academic Literacies. ESP Volume I I, Issue I, 20 I 2.

6 Thaiss, Ch. "The international WACMID mapping project: objectives, methods, and early results". En Bazerman, Ch.; Krut, R.; Lunsford, K.; McLeod, S.; Null, S.; Rogers, P \& Stansell, A. Traditions of Writing Research. New York / London: Routledge, 20 I0, págs. 252-264; Bazerman, Ch.; Bonini, A \& Figueredo, D. (Eds) (Genre in a changing world. Colorado/Indiana: Parlor Press and WAC Clearinghouse, 
en América Latina y en nuestro país el reconocimiento de que tanto quienes se forman para ingresar a la carrera académica ${ }^{7}$ como los estudiantes que transitan sus estudios universitarios necesitan desarrollar habilidades discursivas en textos de los ámbitos académico, cientíico y profesional ${ }^{8}$.

Cabe, sin embargo, postular las razones por las cuales resulta necesaria la creación de un programa de escritura que se sostenga a lo largo de la carrera más que la instalación de acciones puntuales, ya sean éstas cursos paralelos o trabajos al interior de las materias específicas orientados por los profesores que las dictan.

En principio, un programa de las características del PROLEA-UFLO no se plantea, desde la concepción que lo orienta, como la solución a deficiencias de niveles anteriores de enseñanza ni a carencias de los estudiantes. Y esto se debe, fundamentalmente, a que las habilidades de lectura y escritura que puedan adquirirse en la escuela no son suficientes para el desempeño en la Universidad, así como tampoco lo son los cursos introductorios a los estudios universitarios. Tanto el ingreso como el avance en el cursado de una carrera universitaria implican nuevas experiencias, en un nuevo contexto educativo, con prácticas muy diferentes de las conocidas por los estudiantes hasta ese momento. En el abordaje de los contenidos específicos de cada materia del currículum, los estudiantes abordan textos escritos y orales con un uso del lenguaje que les resulta ajeno, un lenguaje que difiere del que es propio de las esferas de comunicación cotidiana y se especializa en ámbitos de formación científica. Por otra parte,

2009; Thaiss, Ch.; G. Bräuer, P. Carlino; Ganobcsik-Williams, L. \& Sinha, A. Writing Programs Worldwide: Profiles of Academic Writing in Many Places. Perspectives on Writing. Fort Collins, Colorado: The WAC Clearinghouse and Parlor Press, 2012.

7 Pereira, C. \& di Stefano, M. "El taller de escritura en posgrado: Representaciones sociales e interacción entre pares". Rev. Signos v.40 (64), 2007, págs. 405-430; Arnoux, E. (Dir.) Escritura y producción de conocimiento en las carreras de posgrado. Buenos Aires: Santiago Arcos, 2009; Carlino, P. La experiencia de escribir una tesis: contextos que la vuelven más difícil. Anales del Instituto de Lingüística, Vol. xxiv-xxv-xxvi: 4I-62, 2005.

8 di Stéfano M. \& Pereira M.C. "La enseñanza de la lectura y la escritura en el nivel superior: procesos, prácticas y representaciones sociales" en: Paula Carlino (Coord) Textos en Contexto: Leer y escribir en la universidad, Asociación Internacional de Lectura, Lectura y Vida, Buenos Aires, 2004, págs. 23-4I; Arnoux, E., Nogueira, S. \& Silvestri, A. "La escritura producida a partir de la lectura de textos polifónicos. Evaluación del desempeño de grupos con diferente entrenamiento lector previo", en M. C. Martínez (comp.) Aprendizaje de la argumentación razonada, Cali, Cátedra UNESCO y Universidad de Cali, 200 I; UNLu. La Lectura y Escritura como prácticas académicas universitarias, Luján, Bs. As: UNLU, Departamento de Educación, 200 I; Moyano, E.I. "Escritura académica a lo largo de la carrera: un programa institucional. Revista Signos 43 (74), 20 I0, págs. 465-488; Adelstein, A. \& Kuguel, I. Los textos académicos en el nivel universitario. Los Polvorines: Universidad Nacional de General Sarmiento, 2004; Carlino, P. Escribir, leer y aprender en la universidad. Una introducción a la afabetización académica. Buenos Aires: F.C.E., 2005; Carlino, P. (Coord.) "Procesos y prácticas de escritura en la educación superior". Signo \& Seña N 16, 2006. Buenos Aires: Facultad de Filosofía y Letras, UBA; Padilla, C. "Argumentación académica: La escritura de ponencias en el marco de una asignatura universitaria", Actas del XI Congreso Nacional de Lingüística, SAL, Santa Fe, Arg.: Univ. Nac. del Litoral, 2009; Parodi, G. Alfabetización académica y profesional en el Siglo XXI: Leer y escribir desde las disciplinas. Santiago de Chile: Ariel, 20 I 0; Moyano, E.I. \& Natale, L. "Teaching Academic Literacy across the University Curriculum as Institutional Policy. The case of the Universidad Nacional de General Sarmiento (Argentina)". En: Thaiss, Ch,; G. Bräuer; P. Carlino; L. Ganobcsik-Williams, and A. Sinha. Writing Programs Worldwide: Profiles of Academic Writing in Many Places. Perspectives on Writing. Fort Collins, Colorado: The WAC Clearinghouse and Parlor Press, 2012; Navarro, F. "Trayectorias de formación en lectura y escritura disciplinar en carreras universitarias de humanidades: diagnóstico y propuesta institucional". Revista Mexicana de Investigación Educativa I8 (58), 20 I3, págs. 709-734; Núñez Cortés, J.A. "Una aproximacion a los centros de escritura en Iberoamérica". Revista Legenda, Vol 17 N¹7, 20 I3, págs. 64- I02; Molina Natera, $\vee$. "Centros de escritura: una mirada retrospectiva para entender el presente y futuro de estos programas en el contexto latinoamericano". Revista Legenda Vol. 18 N¹8, 2014, págs. 9-33. 
ya sea a través de la lectura o la escritura, en el avance de la carrera se van enfrentando con géneros académicos y profesionales que les son también desconocidos: así, tanto el propósito como la estructura y el lenguaje de estos textos se plantean como una barrera para su acceso, y por ende, para el aprendizaje de los contenidos específicos de las asignaturas?.

Así, un nuevo campo de experiencia, constituido por la institución universitaria y los contenidos disciplinares abordados en ella, implican el uso de nuevos géneros y de un lenguaje más abstracto, técnico y elaborado que el utilizado en la experiencia inmediata, del sentido común, esto es, los géneros y el lenguaje de las diferentes disciplinas científicas, según han sido caracterizadas en el marco de la Lingüística Sistémico-Funcional (LSF) ${ }^{10}$. Este hecho, entonces, provoca la necesidad de nuevos aprendizajes. Y, en la educación formal, nuevos aprendizajes demandan nuevos espacios de enseñanza.

En cuanto al tipo de enseñanza que demanda la situación descripta, no se trata de una enseñanza remedial, es decir que solucione problemas no resueltos en instancias anteriores, sino una enseñanza propedéutica, que prepare al estudiante para las nuevas situaciones que debe enfrentar a lo largo de sus estudios universitarios y a las que deberá enfrentar como profesional cuando termine su carrera. Por otra parte, no se trata de la enseñanza que algunos profesores conscientes del problema asuman como necesaria ni de otros dispositivos como talleres de escritura o espacio para tutoreo, sino de una responsabilidad institucional, que implique el trabajo interdisciplinario conjunto entre los profesores de Letras que integran el equipo del Programa y los docentes de las materias específicas de cada carrera que son seleccionadas para su adscripción al Programa"!.

Son las instituciones universitarias las que deben afrontar este desafío, así como sostenerlo a lo largo de la carrera. Esto, como ha sido observado en otras experiencias, obedece a que, por un lado, la incorporación de nuevos géneros se extiende hasta finalizar la carrera; y, por otro, cada género merece ser enseñado hasta que el estudiante adquiera la habilidad de aprender por sus propios medios ${ }^{12}$.

$9 \quad$ Halliday, M. \& Martin, J.R. Writing science: Literacy and discursive power. Pittsburgh: University of Pittsburgh Press, 1993.

I0 Halliday, M. \& Martin, J.R. Writing science: Literacy and discursive power. Pittsburgh: University of Pittsburgh Press, I 993; Martin, J.R. \& Veel, R. (Eds.) Reading science. London: Routledge, 1998; Korner, H.; Mclnnes, D. \& Rose, D. Science literacy. NSW: NSW AMES, 2007; Wignell, P. On the discourse of social science. Darwin: Charles Darwin University Press, 2007; Banks, D. The development of Scientific Writing. Linguistic Features and Historical Context. London: Equinox, 2008; Hood, S. Appraising research: evaluation in academic writing. London: Palgrave Macmillan, 2010 y para el español, Oteiza, T. "Solidaridad ideológica en el discurso de la historia: Tensión entre orientaciones monoglósicas y heteroglósicas". Revista Signos 42 (70), 2009, págs. 21 9-244; Oteiza, T. "Patrones valorativos en el discurso oficial de Derechos Humanos en Chile: dando valor al pasado y construyendo memorias históricas en la sociedad". Discurso y Sociedad 4 (I), 20 I0, págs. I5I- I83; Moyano, E.I. "Argumentación en Economía: Negociación de una Interpretación". Revista R.I.L.L. № I7, vol I y 2, 20 I2; Moyano, E.I. "La Discusión en artículos de Microbiología: género, compromiso y construcción del conocimiento". Revista Onomázein, Número Especial IX ALSFAL, 20 |4, pp. I6 | - |85; Moyano, E.I. "Patrones de realización de la proyección en la Discusión de artículos de investigación producidos en español en dos disciplinas". Revista D.E.L.T.A. 3 I (I), 20I5, págs. I43-I83.

I I Moyano, E.I. "La escritura académica: una tarea interdisciplinaria a lo largo del curriculum universitario". Texturas 4 (4), 2004, págs. 109-120.

12 Moyano, E.I. "Enseñanza de habilidades discursivas en español en contexto pre-universitario: Una aproximación desde la LSF". Revista Signos 40 (65), 2007, págs.573- 608; Moyano, E.l. "Escritura académica a lo largo de la carrera: un programa institucional. Revista Signos 43 (74), 2010 , págs. 465-488. 


\section{Posicionamiento teórico}

La creación de programas de lectura y escritura en las universidades demanda de los docentes una formación profesional sólida con respecto al nuevo objeto a enseñar y a cómo enseñarlo. Esto obliga a reconsiderar el rol docente, las actividades asignadas a los estudiantes y los contenidos sobre el discurso académico y profesional a enseñar. Así, con respecto a este último punto, los estudiantes deberán familiarizarse con los textos y su contexto: su propósito social, su estructura y las características de lenguaje que les son propias, adecuadas al contexto de uso. Este trabajo requiere de una teoría de aprendizaje, una teoría de géneros y una propuesta pedagógica como metodología de enseñanza.

Con respecto a una teoría del aprendizaje, en el PROLEA-UFLO se parte de la propuesta de Halliday ${ }^{13}$, basada en desarrollos previos elaborados en el marco de la LSF. Esta propuesta, además, se liga con la concepción de Vigotsky'4, que entiende que los sistemas semióticos, entre los que se encuentra el lenguaje, son herramientas culturales que configuran la psiquis humana y favorecen el desarrollo cognitivo. A su vez, estos procesos de pensamiento, como señala Wersch ${ }^{15}$, están mediados por géneros discursivos como instrumentos culturales.

Halliday ${ }^{16}$ considera que todo aprendizaje es un proceso de producción de significado. Teniendo en cuenta que el lenguaje es la forma prototípica de la semiosis humana, entonces el desarrollo del lenguaje es al mismo tiempo el desarrollo del aprendizaje: no puede decirse que el lenguaje sea solo un dominio del conocimiento sino que es la condición esencial para el proceso mediante el cual la experiencia se transforma y deviene en conocimiento. Más aun, cuando interviene la escritura, se trata de una reconstrucción de la experiencia que expande el conocimiento y lo profundiza, mediante el uso de abstracciones y metáforas gramaticales ${ }^{17}$.

Aprender a significar es, en consecuencia, aprender a expandir el potencial de significación. Entonces, aprender contenidos es aprender a usar el lenguaje para la producción de esos contenidos. Y si, como ya se señaló, el lenguaje de las disciplinas científicas es un tipo particular de lenguaje, desarrollado especialmente para la producción científica ${ }^{18}$, es necesario favorecer en los estudiantes el desarrollo de estas formas de lenguaje.

I3 Halliday, M.A.K. "Towards a Language-based theory of learning". Linguistics and Education, 5, 1993, págs. 93- I 6.

14 Vigotsky, L. Pensamiento y lenguaje. Buenos Aires: Fausto, 1998.

15 Wertsch, J. Voces de la Mente. Un Enfoque Sociocultural para el Estudio de la Acción Mediada. Madrid: Visor, 1991.

16 Halliday, M.A.K. "Towards a Language-based theory of learning". Linguistics and Education, 5, 1993, pág. 94.

17 Halliday, M.A.K. "Towards a Language-based theory of learning". Linguistics and Education, 5, 1993, págs. I09-I I I.

I8 Halliday, M.A.K. "The construction of Knowledge and Value in the Grammar of Scientific Discourse: Charles Darwin's The Origin of the Species". En Halliday, M. \& J.R. Martin Writing science: Literacy and discursive power. Pittsburgh: University of Pittsburgh Press, 1993, págs. 86-105. 
Por otra parte, la teoría de género elaborada por Martin ${ }^{19}$, que también sustenta la propuesta, supone el concepto de Halliday de contexto intrínseco, es decir, de contexto "encapsulado" en el texto, puesto de manifiesto (realizado) a través de las elecciones lingüísticas efectuadas para la escritura ${ }^{20}$. Teniendo esto en cuenta, Martin y su equipo definieron el concepto de género para fines didácticos como una actividad social dividida en pasos o etapas, con un propósito definido, en el que los hablantes se relacionan como miembros de nuestra cultura ${ }^{21}$. Este concepto implica la realización de la actividad social a través del lenguaje, ya sea que éste funcione como auxiliar o como constitutivo de la acción. Como señalan Martin \& Rose:

En términos de una lingüística funcional [...], los géneros se definen como configuraciones recurrentes de significados $[. .$.$] y estas configuraciones de significados$ representan o llevan a cabo las prácticas sociales de una cultura dada. ${ }^{22}$

Así, las actividades sociales entendidas como género se construyen como significado y se manifiestan en el lenguaje a través de la estructura de los textos y de las elecciones lingüísticas realizadas para construir tres aspectos de la semiosis humana: el campo, entendido como actividad que se lleva a cabo en el texto así como su contenido; el tenor, es decir las relaciones que se establecen entre los participantes de la interacción; y el modo o rol que el lenguaje juega en la construcción del texto, incluyendo el nivel de abstracción utilizado según el campo y el tenor.

En consecuencia, los estudiantes necesitan, al abordar un nuevo género, reflexionar sobre el contexto en el que tiene lugar, su estructura esquemática y los rasgos lingüísticos más relevantes entre los que lo caracterizan.

19 Martin, J.R. "Analyzing genre: functional parameters". En: Christie, F. \& Martin, J.R. (ed). Genre and institutions: Social processes in the workplace and school. London: Continuum, 1997, págs.3-39; Martin, J.R. "Modelling context: a crooked path of progress in contextual linguistics (Sydney SFL)". En Ghadessy, M. (Ed). Text and Context in Functional Linguistics. Amsterdam: Benjamins (CILT Series IV), 1999, págs. 25-61; Martin, J.R. "A context for genre: modelling social processes in functional linguistics". En: Devilliers, J \& R. Stainton (Eds.) Communication in Linguistics: papers in honour of Michael Gregory. Toronto: GREF (Theoria Series I0), 200 I, págs. 287-328; Martin, J.R. "Evolving systemic functional linguistics: beyond the clause". Functional Linguistics, I:3, 2014.

20 Halliday, M.A.K. \& Hasan, R. Language, context, and text: Aspects of language in a social-semiotic perspective. Deakin University Press, 1985; Halliday, M.A.K. El lenguaje como semiótica social. México: FCE, 1982; Halliday, M.A.K. "Computing Meanings: Some Reflections on Past Experience and Present Prospects". En: Computational and Quantitative Studies. Collected Works of M.A.K. Halliday, Vol 6, Editado por Jonathan J. Webster. London: Continuum, 2005, págs. 239-267; Halliday, M.A.K. 'The Notion of 'Context' in Language Education". En: Language in Education. Collected Works of M.A.K. Halliday, Vol 9, Editado por Jonathan J. Webster. London: Continuum, 2007, págs. 269-290.

2I Martin, J.R. "Analyzing genre: functional parameters". En: Christie, F. \& Martin, J.R. (ed). Genre and institutions: Social processes in the workplace and school. London: Continuum, 1997, pág. 13.

22 Martin, J.R. \& Rose, D. Genre Relations: mapping culture. London, Equinox, 2008, pág. 6. Traducción de las autoras. 
Con este fin, la denominada "Escuela de Sydney", constituida por Martin y sus colegas, ha diseñado una propuesta didáctica ${ }^{23}$ que ha sido adaptada al contexto argentino ${ }^{24}$ para proponerla como metodología de enseñanza.

En esta adaptación, Moyano propone la existencia de tres momentos para la enseñanza de géneros (Figura I). El primero, la Deconstrucción, consiste en la reflexión, a partir de textos reales que realicen el género en cuestión, sobre su contexto, su estructura esquemática y algunos rasgos lingüísticos relevantes. En segundo lugar, se propone, en el contexto académico, reemplazar la Construcción Conjunta por el Diseño Conjunto de textos (no considerado en el modelo original), lo que implica reflexionar con los estudiantes sobre la planificación del escrito que tienen que realizar mediante la elaboración de esquemas. La Construcción Conjunta, en términos de escritura conjunta, se utiliza como estrategia en los momentos en que el objeto de enseñanza sea un rasgo de lenguaje determinado, que necesite de este tipo de tarea, como el uso de la metáfora gramatical ${ }^{25}$ o el de proyecciones metafóricas ${ }^{26}$, por ejemplo. Finalmente, una vez que los estudiantes han escrito su propio texto, se realiza la Edición Conjunta, no existente tampoco en el modelo original, que consiste en la deconstrucción de algún o algunos textos producidos por los estudiantes, a fin de guiarlos en un trabajo conjunto para que aprendan a corregir sus escritos en forma independiente cuando éstos presenten problemas o necesiten algún tipo de reformulación.

\begin{tabular}{|l|l|l|l|}
\hline \multirow{2}{*}{\begin{tabular}{l} 
Negociación del Campo \\
\multirow{2}{*}{$\begin{array}{l}\text { Determinación del } \\
\text { contexto }\end{array}$}
\end{tabular}} & \multicolumn{2}{|l|}{ Deconstrucción del género } & $\begin{array}{l}\text { - Lectura conjunta } \\
\text { - Lectura en pequeños grupos } \\
\text { - Lectura individual }\end{array}$ \\
\cline { 2 - 4 } & \multirow{2}{*}{$\begin{array}{l}\text { Diseño del } \\
\text { texto }\end{array}$} & $\begin{array}{l}\text { Construcción } \\
\text { de ejemplares } \\
\text { genéricos }\end{array}$ & $\begin{array}{l}\text { - Escritura conjunta } \\
\text { - Escritura en pequeños grupos } \\
\text { - Escritura individual }\end{array}$ \\
\cline { 2 - 5 } & \multicolumn{2}{|l|}{$\begin{array}{l}\text { Edición de los propios escritos } \\
\text { - Edición conjunta pequeños grupos }\end{array}$} \\
\hline
\end{tabular}

Figura I: Dispositivo didáctico para el desarrollo de la lectura y la escritura basada en géneros discursivos ${ }^{27}$.

23 Martin, J.R. "Modelling context: a crooked path of progress in contextual linguistics (Sydney SFL)". En Ghadessy, M. (Ed). Text and Context in Functional Linguistics. Amsterdam: Benjamins (CILT Series IV), 1999, pp. 25-6I; Martin, J.R. "Genre and language learning: A social semiotic perspective". Linguistics and Education 20, 2009, págs. I 0-21; Rose, D. \& Martin, J.R. Learning to Write, Reading to Learn. Genre, Knowledge and Pedagogy in the Sydney School. UK: Equinox, 2012.

24 Moyano, E.I. "Enseñanza de habilidades discursivas en español en contexto pre-universitario: Una aproximación desde la LSF". Revista Signos 40 (65), 2007, págs. 573- 608; Moyano, E.l. "Deconstrucción y Edición Conjuntas en la enseñanza de la escritura. La reflexión sobre género y discurso en la formación académica y profesional”. En: Anais VI Simpósio Internacional de Estudos de Gêneros Textuais (SIGET), 20II.

25 Halliday, M. "Things and relations: Regrammatizing experience as technical knowledge". In: Martin, Jim; Veel, R. (Eds.) Reading science. London: Routledge, 1998, págs. I85-236.

26 Hood, S. Appraising research: evaluation in academic writing. London: Palgrave Macmillan, 20 I 0; Moyano, E.l. "Proyección congruente y metafórica en las discusiones de artículos científicos de tres disciplinas en español". En: Vian Jr., O. \& Caltabiano, C. (Orgs.) Língua(gem) e suas múltiplas faces. San Pablo: Mercado de Letras, 20 I3, pp. I09-133; Moyano, E.l. "Patrones de realización de la proyección en la Discusión de artículos de investigación producidos en español en dos disciplinas". Revista D.E.L.T.A. 3 I (I), 20 I5, págs. I43- I83.

27 Moyano, E.l. "Enseñanza de habilidades discursivas en español en contexto pre-universitario: Una aproximación desde la LSF". Revista Signos 40 (65), 2007, págs. 573- 608. 


\section{Características del PROLEA-UFLO}

A partir de los lineamientos teóricos y pedagógicos enunciados, el PROLEA-UFLO se concibe como enseñanza de géneros al interior de las materias de la currícula de cada una de las carreras que se dictan en la Universidad: como ya se señaló, no se crean materias especiales para la enseñanza de las habilidades de lectura y escritura ni se asigna la responsabilidad de esta tarea a especialistas en materias específicas de cada carrera, sino que se dispone de tiempo de algunas de las asignaturas específicas para que un profesor del equipo del Programa se haga cargo de enseñar a los estudiantes las prácticas de lectura y escritura requeridas, junto con el docente a cargo de la materia en cuestión.

Esto supone la creación progresiva de un equipo docente en Letras que recibe formación especializada para este tipo de trabajo al tiempo que lo desarrolla. Un miembro de este equipo docente se "asocia" con los docentes de materias específicas de las diferentes carreras, a fin de llevar adelante, de manera conjunta, la enseñanza de la lectura y la escritura basada en géneros ${ }^{28}$.

Se conforman así equipos interdisciplinarios, que llevan adelante diversas acciones. La primera de ellas se denomina Negociación entre Pares, y consiste en el establecimiento de una serie de acuerdos para la enseñanza. Estos acuerdos incluyen la definición del o los géneros que el profesor de la materia pretende que sus estudiantes escriban y su rol en el proceso de aprendizaje; caracterizar la estructura esquemática de esos géneros; establecer algunas características discursivas a enseñar; establecer la proximidad de los géneros demandados con los profesionales o científicos y explicar la inclusión de pasos para su adaptación a la universidad cuando esto ocurra; acordar las consignas de lectura y de escritura así como los modos de aplicación de la propuesta didáctica y elaborar pautas de evaluación, incluyendo la elaboración de rúbricas y de un cronograma, que serán conocidos también por los estudiantes ${ }^{29}$.

Este trabajo interdisciplinario se vuelve sumamente útil para el proceso y enriquecedor para los profesionales que participan de él. Efectivamente, los profesores de materias específicas a las diferentes carreras conocen el campo de su disciplina así como el de la actuación profesional, pero, en la mayoría de los casos, tienen escasa conciencia lingüística y genérica, de manera que no tienen los recursos necesarios para caracterizar los géneros que esperan que sus estudiantes produzcan ni disponen de recursos para su enseñanza ${ }^{30}$. Hay, desde luego, excepciones a esta generalización ${ }^{31}$.

28 Moyano, E.I. "La escritura académica: una tarea interdisciplinaria a lo largo del currículum universitario". Texturas 4 (4), 2004, pp. 109-120; Moyano, E.I. "Escritura académica a lo largo de la carrera: un programa institucional. Revista Signos 43 (74), 20 I0, págs. 465-488.

29 Moyano, E.I. "Escritura académica a lo largo de la carrera: un programa institucional. Revista Signos 43 (74), 20 I 0, págs. 465-488.

30 Moyano, E.l. "Negotiating Genre: Lecturer's Awareness in Genre Across the Curriculum Project at the University Level". In: Bazerman, Charles, Bonini, Adair, and Figueiredo, Débora. 2009. Genre in a Changing World. Perspectives on Writing, 2009, págs. 449-464. Fort Collins, Colorado: The WAC Clearinghouse and Parlor Press.

31 UNLu. La Lectura y Escritura como prácticas académicas universitarias, Luján, Bs. As: UNLU, Departamento de Educación, 200 I; Carlino, P. Escribir, leer y aprender en la universidad. Una introducción a la afabetización académica. Buenos Aires: F.C.E., 2005; Vázquez, A.; Jakob, l.; Novo, M.C. \& Pelizza, L. (Comp.) Lectura, escritura y aprendizaje disciplinar. Río IV: UniRío Editora, Universidad Nacional de Río Cuarto, 2012. 
Por otra parte, los docentes de Letras se capacitan en la teoría lingüística que sustenta el Programa, tienen la capacidad profesional para la descripción de géneros y formación para la enseñanza de la lectura y la escritura, además de que reciben formación con respecto a la propuesta pedagógica, a la que pueden adaptarse rápidamente. Carecen, sin embargo, del conocimiento del campo y de sus géneros, especialmente cuando se trata de géneros profesionales, más aun cuando se trata de lo que pueden considerarse, siguiendo a Swales ${ }^{32}$, géneros ocultos. Para el trabajo de la caracterización de estos géneros, se ha diseñado un dispositivo que se lleva a cabo en el marco de la Negociación entre Pares ${ }^{33}$.

Luego de la Negociación entre Pares, en el momento acordado para la asignación de la tarea de escritura a los estudiantes, el profesor de Letras dicta una clase de Deconstrucción, que implica un trabajo de lectura conjunta con identificación de los rasgos centrales del texto, según ya se explicó. A partir de allí, se propone la caracterización del género a producir y se los guía a los estudiantes para el Diseño del texto. Luego del período asignado para la tarea de escritura, el profesor de Letras recibe los trabajos y selecciona un trabajo completo o fragmentos de varios que presenten problemas que se hayan detectado como comunes a la mayoría del grupo. En la siguiente clase asignada, se hace la tarea de Edición Conjunta, que consiste en discutir con los estudiantes si el texto elegido realiza el género en su estructura esquemática y si presenta los rasgos de lenguaje enseñados. Para ello se sigue la rúbrica diseñada por los docentes en conjunto, que ya ha sido socializada con los estudiantes luego del período de Deconstrucción y Diseño conjuntos. Asimismo, se discute si existen en los textos seleccionados segmentos de difícil comprensión, para consensuar con todo el grupo su re-elaboración. De esta manera, los estudiantes aprenden también a editar textos, lo que aplicarán luego a la revisión de los suyos.

Un Programa de estas características resulta ventajoso en relación con otras modalidades de enseñanza de géneros académicos ya ensayados con anterioridad. Con respecto a los talleres de escritura paralelos a las materias específicas del currículum, se ha notado que la enseñanza a lo largo de la carrera permite contextualizar mejor los géneros y la tarea de escritura, de manera que el estudiante puede comprender con más claridad la vinculación entre las tareas propuestas y su formación como estudiante y como futuro profesional, realizando transferencia de aprendizaje entre diferentes situaciones ${ }^{34}$. Hyland ${ }^{35}$ ha señalado, en cambio, cómo los talleres paralelos son vividos como tareas apartadas de la formación disciplinar, de manera tal que los estudiantes los disocian de ella. En cuanto a la enseñanza al interior de las materias pero a cargo de profesores de materias específicas, como

32 Swales, J. "Occluded Genres in the Academy: The Case of the Submission Letter". En: Ventola, E. \& Mauranen, A. (Eds.) Academic Writing. Intercultural and Textual Issues. Amsterdam: Benjamins, 1996, págs. 45-58.

33 Moyano, E.l. "Hacia la caracterización de géneros profesionales: algunas reflexiones teórico-metodológicas". En: Nothstein, S.; Pereira, M.C. \& Valente, E. (Comps) Libro de Actas del Congreso Regional de la Cátedra UNESCO en Lectura y Escritura: "Cultura Escrita y Políticas Pedagógicas en las Sociedades Latinoamericanas Actuales". Los Polvorines: Universidad Nacional de General Sarmiento, 2012, págs. I567-I586.

34 Giudice, J. \& Moyano, E. "Apropiación del discurso de la economía: Análisis evolutivo de un caso". En: Barbara, L. \& Moyano, E. (Eds.), Textos y lenguaje académico. Exploraciones sistémico-funcionales en portugués y español. Los Polvorines/San Pablo: UNGSPUCSP, 2011, págs. 91-112.

35 Hyland, K. Teaching and Researching Writing. London: Longman, 2002. 
propone Carlino ${ }^{36}$, se ha mencionado ya que en la mayoría de los casos estos docentes no tienen la formación necesaria para la caracterización de géneros ni tampoco disponen de estrategias didácticas adecuadas para su enseñanza. Por otra parte, muchos de ellos consideran que esta tarea les es ajena, mientras que, luego de una buena negociación aceptan participar del trabajo acompañados de un socio de enseñanza: un profesor de Letras especializado. Para que esta modalidad tenga buenos resultados, se requiere de ciertas condiciones que no es posible detallar aquî ${ }^{37}$.

En la Universidad de Flores, este diseño se encuentra en proceso de aplicación en cada carrera, a lo largo del currículum, iniciando en el primer cuatrimestre de primer año y llegando hasta el último cuatrimestre de la carrera. Por ahora, se ha resuelto asociar una materia por año de cursado al Programa, con la expectativa de llegar, una vez abarcadas todas las carreras de la Universidad, a una materia por cada semestre en cada carrera. Hasta el momento, y a partir de una prueba piloto que se desarrolló entre 2013 y 20 I4, se está trabajando en las carreras de Psicología, Psicopedagogía, Profesorado en Educación Física, Derecho, Ingeniería Ambiental y Seguridad, Higiene y Control Ambiental.

\section{Algunos resultados en el inicio de los estudios universitarios}

Con el objetivo de ilustrar el modo de implementación del Programa, se seleccionó el caso de la segunda experiencia en una asignatura del primer cuatrimestre del primer año de la Licenciatura en Psicología. Con este objetivo, se ejemplificará la evolución observada en los escritos de los estudiantes analizando borradores iniciales y sus correspondientes versiones finales.

La materia fue cursada por 150 estudiantes distribuidos en tres turnos, durante cuatro horas semanales (68 horas en total). En cuanto a las características generales del alumnado, se debe señalar la existencia de dos grupos diferenciados en cuanto a formación y franja etaria. Existe un mayoritario grupo de jóvenes de entre 18 a 20 años, de reciente egreso de la escuela media, y otro, minoritario, de adultos con título terciario o universitario, que suelen iniciar sus estudios para darle cauce a una vocación postergada. Este sector tiene una participación activa en el aula, y aunque por lo general son más competentes que sus compañeros más jóvenes, también manifiestan problemas a la hora de escribir un texto. Así esta asignatura transcurre en el difícil momento en que la mayoría de los estudiantes está haciendo el esfuerzo por incorporarse a la comunidad discursiva académica, cuyas prácticas de lectura y de escritura desconoce ${ }^{38}$.

36 Carlino, P. Escribir, leer y aprender en la universidad. Una introducción a la afabetización académica. Buenos Aires: F.C.E., 2005.

37 Moyano, E.I. "La escritura académica: una tarea interdisciplinaria a lo largo del curriculum universitario". Texturas 4 (4), 2004, págs. I09-120; Braidot, N.; Moyano, E.; Natale, L. \& Roitter, S. "Enseñanza de la lectura y la escritura como política institucional a lo largo de las carreras de ingeniería del IDEI-UNGS". En: Trabajos completos, 2008, Cód. 368. VI CAEDI, UNSa-UCASALCOPAIPA. Salta, septiembre 2008.

38 Swales, J. Genre analysis. English in academic and research settings. Cambridge: Cambridge University Press, 1990. Como referencia en el contexto argentino, véase entre otros: Arnoux, E., di Stefano, M. \& Pereira, C. La lectura y la escritura en la universidad. Buenos Aires: EUDEBA, 2002. 
Es necesario destacar que los alumnos de la Universidad de Flores ingresan en forma directa a la carrera, sin curso de ingreso, taller de aprestamiento o nivelador que se aboque a la enseñanza de habilidades de lectura y escritura. Cabe además recordar las dificultades en la comprensión y producción de textos detectadas en los estudiantes de escuela media (el país obtuvo un rendimiento muy bajo en el área de Lengua en las últimas pruebas PISA ${ }^{39}$ ), lo que permite inferir su situación al finalizar esa etapa e ingresar a la universidad. Por otro lado, diversas investigaciones diagnósticas han revelado problemas en el ingreso a los estudios superiores así como a lo largo de la carrera universitaria en distintas instituciones, que se repiten en el alumnado que cursa la materia en cuestión: comprensión lectora deficiente, escasa habilidad para la producción de textos orales y escritos, dificultades para seleccionar contenidos disciplinares pertinentes para la escritura de un texto, para integrar diversas voces o confrontar posiciones o para argumentar ${ }^{40}$. En este sentido, existe consenso en la necesidad de destinar recursos institucionales para la enseñanza de la escritura de textos académicos y profesionales en la universidad, como ya se señaló antes.

\section{La negociación entre pares}

Para la implementación del PROLEA en la asignatura seleccionada por la dirección de la carrera, se estableció como momento privilegiado de intervención la asistencia para la realización de un trabajo práctico final. La primera tarea, entonces, fue precisar y acordar con los docentes el género discursivo en el marco del cual los estudiantes deberían inscribir su texto. Se aconsejó ubicar la consigna en una

39 Cf. TN, 3/12/20/3, disponible en: http://tn.com.ar/sociedad/prueba-pisa-el-texto-que-la-mitad-de-los-alumnos-argentinos-nocomprendio_425499

40 De Lelia C. \& Ezcurra, A. M. (Dir). Competencias cognitivas de alumnos del penúltimo año de educación media. Instituto de Estudio Acción Social. Investigación destinada a la Universidad Nacional de General Sarmiento, 1994; Ezcurra, A. (Dir). Competencias cognitivas de alumnos de primer ingreso universitario potencial (en actividades de comprensión y producción de textos de tipo expositivo y argumentativo de género académico). Los Polvorines: Universidad Nacional de General Sarmiento Investigación de la Unidad Pedagógica Universitaria, 1995; di Stéfano M. \& Pereira M.C. "Representaciones sociales de la lectura en el proceso de comprensión lectora", Signo \& Seña № 8, 1997, Perspectivas en Psicolingüística: modelos, objetos y métodos. Instituto de Lingüística, UBA, págs. 3 I 8-340; di Stéfano M. \& Pereira M.C. "La enseñanza de la lectura y la escritura en el nivel superior: procesos, prácticas y representaciones sociales" en: Paula Carlino (Coord) Textos en Contexto: Leer y escribir en la universidad, Asociación Internacional de Lectura, Lectura y Vida, Buenos Aires, 2004, págs. 23-4I; Arnoux, E., Nogueira, S. \& Silvestri, A. "La escritura producida a partir de la lectura de textos polifónicos. Evaluación del desempeño de grupos con diferente entrenamiento lector previo", en M. C. Martínez (comp.) Aprendizaje de la argumentación razonada, Cali, Cátedra UNESCO y Universidad de Cali, 200 I; Arnoux, E., di Stefano, M. \& Pereira, C. La lectura y la escritura en la universidad. Buenos Aires: EUDEBA, 2002; UNLu. La Lectura y Escritura como prácticas académicas universitarias, Luján, Bs. As: UNLU, Departamento de Educación, 200 I; Zalba, E.M. "La palabra interdicta: problemas de producción discursiva en estudiantes universitario". Ponencia presentada en el Simposio Internacional Lectura y escritura: nuevos desafíos, Facultad de Ciencias Políticas y Sociales \& Facultad de Filosofía y Letras de la Universidad Nacional de Cuyo. Mendoza, mayo de 2002; Cubo de Severino, L. "Evaluación de estrategias retóricas en la comprensión de manuales universitario". RILL, No I5, 2002, págs. 69-84; Uslenghi de Murga; Padilla, C.; Ameijide, M.D. \& Douglas, S. "Discurso estudiantil: Representaciones acerca de las competencias discursivas. Análisis crítico del discurso estudiantil en el nivel universitario. Oralidad, lectura y escritura: categorización de dificultades." RILL NI5, 2002, págs. 85- I03; Moyano, E.I. Evaluación Diagnóstica Sumativa del Taller de Lecto-escritura del curso de aprestamiento Universitario de la UNGS, Informe Final de Resultados. Los Polvorines: Publicación interna Secretaría Académica, IDH-UNGS, 2003; Moyano, E.I. "Enseñanza de habilidades discursivas en español en contexto pre-universitario: Una aproximación desde la LSF”. Revista Signos 40 (65), 2007, págs. 573- 608. 
situación comunicativa del ámbito académico: unas jornadas disciplinares para las cuales los alumnos debían elaborar una ponencia que tuviera como tema una breve investigación acerca de un tema abordado en la materia, para exponer oralmente en pocos minutos. Esta investigación implicaba analizar desde diferentes perspectivas teóricas las entrevistas realizadas a un grupo de adultos mayores acerca de su concepción de "felicidad".

Se caracterizó el género elegido, estableciendo los distintos pasos para la estructuración de la ponencia y se hizo una breve descripción funcional de las distintas unidades textuales menores o fases (obligatorias u optativas) que la integran. También se dejó constancia de pautas o requisitos formales y fecha de entrega. Se ofreció, además, una guía para la preparación de la exposición oral de los estudiantes y una lista de materiales de apoyo para la resolución de dudas idiomáticas, problemas gramaticales, estilos de citación. Finalmente, y como producto de un paso fundamental de la negociación entre pares, se incluyó un cronograma de intervenciones del PROLEA.

En el segundo año de implementación del Programa, la guía para la elaboración del texto solicitado y el cronograma a seguir estuvieron disponibles para los estudiantes desde el primer día de clases junto con el programa de la asignatura - en el cual se indicó la asociación con el PROLEA-. De esta manera, quedaron establecidos los requisitos de aprobación desde el inicio.

Dado que en el primer año de implementación del Programa algunos alumnos produjeron textos que fueron considerados modelos genéricos válidos, se seleccionaron específicamente dos para deconstruir con los estudiantes. Estos ejemplares manifestaban modos de organización diferentes, pero ambos válidos como modelo: uno desarrollaba las teorías que se aplicarían luego al análisis del caso concreto; otro adoptaba el camino inverso, esto es, presentar cada caso particular para luego analizarlo según el marco conceptual elegido.

\section{La clase de deconstrucción de un modelo genérico}

En este encuentro el profesor de la materia presentó la guía para la elaboración del texto y la profesora del PROLEA colaboró precisando el tipo de enunciador y lector que se proponía construir así como el registro adecuado para la situación comunicativa. Se realizó una lectura conjunta de fragmentos previamente seleccionados de los modelos genéricos. Por medio de preguntas se orientó a los alumnos para que observaran cómo los textos utilizaban modos de exposición alternativos y validados por el conocimiento científico y se señalaron semejanzas y diferencias. Se los guió en la identificación de los pasos y fases del género, se focalizó en realizaciones lingüísticas adecuadas. Se identificaron algunos recursos que caracterizan al lenguaje científico: lenguaje no congruente, alto grado de abstracción y tecnicidad, metáforas gramaticales, particular uso de la valoración ${ }^{41}$. También

4I Halliday, M. \& Martin, J.R. Writing science: Literacy and discursive power. Pittsburgh: University of Pittsburgh Press, I993; Halliday, M. "Things and relations: Regrammatizing experience as technical knowledge". En: Martin, Jim; Veel, R. (Eds.) Reading science. London: Routledge, 1998, págs. I85-236; Wignell, P. On the discourse of social science. Darwin: Charles Darwin University Press, 2007; Hood, S. Appraising research: evaluation in academic writing. London: Palgrave Macmillan, 2010. 
se repasaron los recursos para distinguir el discurso ajeno y se señaló cómo la ciencia construye un discurso marcadamente heteroglósico, a partir del cual se construye el conocimiento ${ }^{42}$.

Al mismo tiempo que observaron las características del tipo de texto que debían producir, los alumnos pidieron precisiones acerca del marco teórico de la asignatura y de la metodología de análisis, de manera que el profesor de la materia se involucró en la presentación del trabajo y participó en los acuerdos establecidos. Se aprovechó esta instancia para explicitar los objetivos y la modalidad de trabajo del PROLEA: envío de borradores, clases de edición conjunta, acompañamiento del Programa hasta la entrega del trabajo final.

\section{Los primeros borradores: análisis de algunos ejemplos}

Los trabajos recibidos en primera instancia pusieron en evidencia que, en términos generales, los estudiantes poseen grandes dificultades para producir textos como el requerido. Manifestaron una errónea percepción de la situación comunicativa, serios problemas en la adecuación al género y al registro, con escaso control de la valoración. Fue mayoritaria la utilización de un tipo de lenguaje congruente -por momentos coloquial, muy alejado del académico y disciplinar-, con problemas en el nivel léxicogramatical, y en ocasiones, en el uso de normas gramaticales básicas. Como las principales dificultades se concentraron en el paso "Introducción", se seleccionaron las de dos trabajos (en adelante, Caso I y Caso 2), a modo de ejemplo de esta etapa. Con el objetivo de ordenar la exposición, se los analizará por tipo de problema observado, trabajo que también se realizó en la clase de Edición Conjunta.

\section{Inadecuada percepción de la situación comunicativa}

A pesar del instructivo suministrado y del modelo genérico visto en clase, los primeros borradores no se adecuaron a la situación comunicativa planteada, tal como puede observarse en el siguiente ejemplo, del cual se analizarán algunos fragmentos.

Caso I - Primera versión (transcripción textual) ${ }^{43}$

El trabajo que desarrollaremos se centra en un concepto muy amplio, "la felicidad". Sabemos que no es sencillo de definirlo, ya que las personas, por nuestra singularidad, podemos ser felices por diferentes motivos. Es decir, lo que puede generar un estado de felicidad en algunas personas, puede no generarlo en otras. ¿Podríamos aventurarnos a decir que la felicidad va a depender de la percepción que tenga cada ser humano frente a determinados hechos o situaciones que acontecen cotidianamente y/o a lo largo de su vida? (...)

42 Martin, J.R. \& White, P.R.R. The language of evaluation. Appraisal in English. London, Palgrave, 2005.

43 La trascripción de esta cita como de las restantes provenientes de textos de los alumnos es literal, por lo cual los errores ortográficos y/o gramaticales son los que figuran en el original. Las citas se reproducen con expresa autorización de los alumnos. 
En esta oportunidad la investigación la haremos indagando a través de entrevistas a un grupo etario mayor de 60 años de ambos sexos. Posteriormente de un grupo de diez entrevistados, se seleccionarán cinco para aplicar la Escala de Okford. El objetivo es cotejar si dichas personas, han tenido un estilo de vida en concordancia con las descripciones realizadas para alcanzar la felicidad, por los diferentes autores leídos y aportados por la cátedra.

Consideramos que es un trabajo importante para nuestra carrera, dado que recabando datos concretos de experiencias vividas, podríamos en un futuro orientar a quienes lo necesiten, acerca de una calidad de vida que dependerá en gran medida, de los niveles de felicidad y optimismo con que hayan transitado sus caminos. Se trata como mencionamos anteriormente de un trabajo empírico.

Los destinatarios somos nosotros mismos y nuestros compañeros de cursada, porque a partir de las experiencias que expondremos en el desarrollo del trabajo, compartiremos con todos ellos y los profesores, las conclusiones más relevantes a las que arribaremos, intentando dar respuesta a las preguntas que nos formulamos. De este modo apuntamos a enriquecer el saber, articulando la teoría con la práctica.

Es interesante observar que esta introducción, aunque relativamente bien escrita en términos de normativa básica, es totalmente inadecuada. En primer lugar, se parte de una perspectiva errónea del tipo de situación comunicativa planteada y el tipo de actividad a realizar. En vez de un informe de investigación destinado a ser compartido en unas jornadas, se observa en el primer párrafo el planteamiento de una reflexión personal acerca de una temática amplia (la felicidad, el tema propuesto y trabajado en clase), para lo cual se formulan supuestas preguntas orientadoras que exceden por completo los objetivos planteados por la cátedra e incluso las posibilidades de respuesta para alumnos del nivel:

El trabajo que desarrollaremos se centra en un concepto muy amplio, "la felicidad". Sabemos que no es sencillo de definirlo (...) ¿Podríamos aventurarnos a decir que la felicidad va a depender de la percepción que tenga cada ser humano frente a determinados hechos o situaciones que acontecen cotidianamente y/o a lo largo de su vida? (...)

La desorientación en cuanto al alcance y tipo de trabajo se observa en el párrafo destinado a presentar la investigación. En vez de anunciarlo como el objetivo primordial, aparece como una etapa dentro del contexto difuso planteado en el primer párrafo (la reflexión acerca de qué es la felicidad): "En esta oportunidad la investigación la haremos indagando a través de entrevistas a un grupo etario mayor de 60 años de ambos sexos...".

También se presentan objetivos no solicitados y excesivos: "El objetivo es cotejar si dichas personas, han tenido un estilo de vida en concordancia con las descripciones realizadas para alcanzar la felicidad (...)". 
Los mismos problemas se reflejan en la construcción del enunciador y el destinatario adecuado a la situación comunicativa: "Los destinatarios somos nosotros mismos y nuestros compañeros de cursada $(\ldots)^{\prime \prime}$.

\section{Uso de un tipo de lenguaje cotidiano, alejado del disciplinar o académico}

El lenguaje utilizado es el cotidiano, primero en ser desarrollado por todo hablante, más ligado a la oralidad y a la experiencia del mundo de la experiencia, propio del sentido común. Este tipo de lenguaje, denominado congruente, se caracteriza, en líneas generales, por presentar a personas como participantes que afectan por medio de procesos (acciones) a otras personas. En los ejemplos que se transcriben a continuación, se indican con subrayado: "algunas personas", o el sujeto desinencial "nosotros" repuesto entre corchetes, que afecta a otras personas ("a quienes lo necesiten"). Los procesos, dado el campo que se construye en el texto, son principalmente relacionales causales ("generar") y mentales ("consideramos", "recabando", "orientar"), en general modelizados ("puede generar", "podríamos... orientar"), y se realizan a través de verbos en voz activa. Las relaciones lógicas se dan entre cláusulas, realizadas a través de conjunciones - como en "dado que", "porque" señalados con cursiva-.

(...) las personas, por nuestra singularidad, podemos ser felices por diferentes motivos. Es decir, lo que puede generar un estado de felicidad en algunas personas, puede no generarlo en otras [personas].

[Nosotros] Consideramos que es un trabajo importante para nuestra carrera, dado que recabando datos concretos de experiencias vividas, [nosotros] podríamos en un futuro orientar a quienes lo necesiten

Los destinatarios somos nosotros mismos y nuestros compañeros de cursada, porque a partir de las experiencias que expondremos en el desarrollo del trabajo, compartiremos con todos ellos y los profesores (...).

El lenguaje de la ciencia y el académico, en cambio, se caracterizan por ser no-congruentes: presentan como característica primordial el uso de la metáfora gramatical que consiste en que los procesos se realizan como objetos a través de sustantivos (a través del recurso de la nominalización), y las relaciones lógicas se dan dentro de la cláusula, realizadas a través de verbos y de construcciones prepositivas. Un caso particular de metáfora gramatical es la que se da en la función interpersonal, en la que una apreciación personal (del tipo "creo/considero que") puede ser realizada a través de una oración impersonal del tipo "se puede considerar"44.

Lejos de las características típicas del discurso disciplinar o académico, que controla la valoración a través de procesos de despersonalización u objetivación como el arriba señalado, en este caso se observa escaso control de ese rasgo. Además del uso de la primera persona, se expresan explícitamente

44 Halliday, M.A.K. (1994) An Introduction to Functional Grammar, 2nd ed. London: Arnold. 
valoraciones (subrayadas): "Sabemos que no es sencillo de definirlo..."; "¿Podríamos aventurarnos a decir...?"; "nos preguntamos si es viable..."; "podríamos en un futuro orientar..."; "Consideramos que es un trabajo importante para nuestra carrera...", "apuntamos a enriquecer el saber...".

\section{Ausencia de fases obligatorias dentro del paso Introducción}

Aunque en la clase de deconstrucción del modelo se puso énfasis en la observación de las fases obligatorias, fueron notorias las dificultades en este aspecto. Baste el segundo caso, que se transcribirá en forma textual, como ejemplo para el análisis.

\section{Caso 2 - Primera versión (transcripción textual)}

Realizada una breve investigación acerca de qué es la felicidad, en nuestro contexto actual para adultos mayores de 60 años, buscamos relacionar los datos empíricos a modo de lograr una adecuada comprensión de las teorías y observar sus posibles límites.

En el marco de diez entrevistas a adultos mayores de 60 años, seleccionamos y analizamos cuatro entrevistas, a nuestro criterio, más significativas; especificando en cada caso el criterio de selección.

Si bien el tema con respecto a la felicidad se encuentra abordado infinitas veces, en esta oportunidad buscamos reafirmar y ampliar la mirada frente a las diferentes posibilidades y formas que encuentran las personas de esta categoría para ser felices.

En este caso, se puede observar que la estructura de la Introducción no presenta las partes funcionales propuestas en la Deconstrucción. En efecto, el primer párrafo no colabora en presentar el tipo de texto que se ha producido (no define el género discursivo), ni aclara el contexto que motivó la investigación (la rama de la disciplina y el análisis de un tema a través de los diferentes modelos estudiados). De una manera difusa se mencionan objetivos del trabajo ("relacionar los datos empíricos a modo de lograr una adecuada comprensión de las teorías y observar sus posibles límites") pero sin presentar adecuadamente el marco teórico ni justificar de alguna manera su selección. Esta misma desorientación se observa en el segundo párrafo del texto: se confunde marco teórico con metodología ("en el marco de diez entrevistas"), la cual, a su vez, no aparece con claridad. En el tercer párrafo se observa un problema común con el caso anterior: una errónea presentación del tema general. En efecto, se focaliza en las personas entrevistadas y no en las teorías que se abordaron como enseñanza de la disciplina ("buscamos reafirmar y ampliar la mirada frente a las diferentes posibilidades y formas que encuentran las personas..."). Esto es, se desliza hacia la apreciación subjetiva del grado de felicidad o infelicidad de los entrevistados, y no se focaliza, como se indicó en el instructivo, en el análisis de acuerdo a ciertas teorías que deberían haber sido introducidas. 


\section{Comprobación de dificultades en el nivel léxico-gramatical}

En los primeros borradores se pudieron observar problemas en este nivel, que pueden ser ejemplificados con el Caso 2. En efecto, hay un incorrecto uso de verbo des: nótese el problemático uso del participio al comienzo del texto ("Realizada una breve investigación...."); y más adelante, el inadecuado uso del gerundio ("especificando en cada caso...").

Como otro aspecto, se puede comprobar que a pesar de su brevedad, el texto resulta redundante: obsérvese la reiteración léxica de "adultos mayores de 60 años", "entrevistas", "criterio".

Por último, se manifiesta un desorden y complejización excesiva de la sintaxis, tal vez en el intento de emular un registro que se asume erróneamente como más formal o académico. Baste como ejemplo este fragmento, en el cual se subraya la acumulación de infinitivos que aparecen problemáticamente conectados (indicado con cursiva): "buscamos relacionar los datos empíricos a modo de lograr una adecuada comprensión de las teorías y observar sus posibles límites".

\section{Las clases de Edición Conjunta de borradores}

Se realizaron actividades de Edición Conjunta de borradores en sucesivos encuentros (de dos a tres, según cada curso). Estas intervenciones, consideradas un momento fundamental en la implementación del Programa, se realizaron en el aula, en presencia del profesor de la materia, quien colaboró en la clarificación de conceptos teóricos, por lo cual se trató de una tarea en equipo, transdisciplinar.

En esos encuentros la profesora del Programa trabajó con ejemplos previamente seleccionados de los borradores de acuerdo a la siguiente metodología. Se proyectaron fragmentos de borradores enviados (omitiendo datos personales) y se solicitó a los alumnos que colaboraran en una revisión de los textos teniendo en cuenta lo señalado en el instructivo compartido. Entre todos, se subrayaron aciertos y se anotaron en el pizarrón los aspectos a modificar o tomar en cuenta para una edición de los textos. Se re-leyeron indicaciones dadas en el instructivo, se revisaron fragmentos del modelo genérico, se los comparó con los casos seleccionados. Se negociaron aspectos a considerar para una autocorrección y se los ejemplificó con una edición, en forma conjunta, de los borradores. Luego, se solicitó a los alumnos que revisaran sus propios escritos aplicando lo visto. Se aconsejó re-enviar el borrador una vez finalizada la rutina de Edición Independiente.

Como apoyo, la docente del Programa elaboró luego de la primera clase de Edición Conjunta un nuevo instructivo que recuperó lo acordado con los alumnos para facilitarles la tarea de edición. Finalmente, se realizó a través de correo electrónico una devolución individual del último borrador una semana previa a la entrega definitiva, con indicaciones precisas para la edición del texto.

De esta manera, los alumnos tuvieron la oportunidad de escribir y re-escribir de acuerdo a las indicaciones dadas la ponencia solicitada y concluir el proceso de manera satisfactoria en casi todos los casos. Hubo un alto grado de participación, y se pudo observar que incluso los alumnos que habían comenzado con grandes dificultades, lograron adecuar la escritura a las pautas solicitadas. 
En la entrega definitiva, los alumnos colocaron los borradores previos y la corrección realizada por el PROLEA para que el profesor de la asignatura, encargado de ponderar con nota numérica, observara el proceso de aprendizaje y evolución. En todos los casos, la calificación obtenida en este trabajo fue notablemente mejor que la de los exámenes parciales, lo que colaboró con una apreciación positiva del Programa por parte de los alumnos.

\section{Evolución de los textos: avances observados}

Para ejemplificar los avances logrados en esta implementación del Programa, se transcribirán las versiones finales de los casos tomados como ejemplo, para analizarlos.

Caso I, versión final (transcripción completa) ${ }^{45}$ :

El presente trabajo consiste en un primer informe de una breve investigación, acerca de qué es la felicidad para adultos mayores de 60 años. El concepto de felicidad es muy amplio, lo que puede generar un estado de felicidad en algunas personas, puede no generarlo en otras. ¿Es posible aventurarse a decir que la felicidad va a depender de la interpretación, que cada ser humano tiene frente a hechos o situaciones que acontecen a lo largo de sus vidas?

Para realizarlo se tuvieron en cuenta las investigaciones, aportes y avances de la Psicología de la salud. Se han tomado en consideración los autores Tal Ben- Shahar (2008), Martín Seligman (2003) y Víctor Frankl (1989).

En esta oportunidad, el trabajo se llevó a cabo mediante entrevistas con un cuestionario fijo, realizado a diez adultos mayores cuyo rango de edad es de 60 a 87 años, de ambos sexos, en nuestro contexto actual.

De los diez casos relevados se analizarán tres. El criterio de selección se debe a que pese a un común denominador hallado en los diez, en los relatos de éstas personas se ha podido observar, que parecen haber encontrado la felicidad en virtud de otros aspectos. A partir de ello se arribará a conclusiones.

Con respecto al primer borrador ya analizado, se puede observar una evolución favorable de esta versión en varios aspectos. En efecto, cada párrafo realiza las diversas fases o unidades textuales funcionales menores que caracterizan al género y al paso "Introducción": en el primero se presenta el tipo de trabajo y el tema general abordado; en el segundo el marco teórico; en el tercero la metodología y una anticipación de las partes del texto.

45 Por cuestiones de espacio, se aprovecha la transcripción de las versiones finales para focalizar avances. En este ejemplo, una mayor adecuación a la situación comunicativa con doble subrayado; con subrayado simple uso de un lenguaje menos congruente; con negrita y cursiva recursos para controlar la valoración. 
En el primer párrafo se introdujo una oración inicial (indicada con doble subrayado) que sitúa a la comunicación en el contexto adecuado y presenta claramente el género discursivo: "un primer informe de una breve investigación".

Por otra parte, recursos como la nominalización o uso de sustantivos abstractos (indicados con subrayado simple) colaboran en acercar esta versión a un tipo de lenguaje menos coloquial, más académico y disciplinar. En efecto, se puede observar que ya no se trata exclusivamente de "personas" que afectan por medio de procesos materiales a otras personas, sino que aparecen cláusulas donde los participantes son conceptos (el concepto de felicidad, las teorías del campo) vinculados con otros conceptos (el trabajo de investigación).

Este mismo avance se observa en el uso de la valoración, que aparece controlada: al abandono de la primera persona gramatical de la versión inicial por el exclusivo uso de la tercera se le suman recursos como la pasivización ("se llevó a cabo", "se ha podido observar") que colaboran con la impersonalización que caracteriza al discurso académico.

Todos estos recursos permiten, a su vez, construir un tipo de enunciador y de destinatario adecuado a la situación comunicativa planteada desde el inicio: un informe de una investigación del campo disciplinar presentada como ponencia para una jornada.

Caso 2: versión final (transcripción textual completa) ${ }^{46}$

En el presente trabajo se dará cuenta de una breve investigación acerca de qué es la felicidad, dentro de la materia Psicología de la salud de la Licenciatura en Psicología de la UFLO. En nuestro contexto actual para adultos mayores de 60 años, buscamos relacionar los datos empíricos a modo de lograr una adecuada comprensión de las teorías que abordan la temática y que ponen el acento en fortalezas, virtudes, recursos cognitivos, emocionales, vinculares y sociales, y observar sus posibles límites.

En diez entrevistas a adultos mayores de 60 años, seleccionamos y analizamos tres, a nuestro criterio, más significativas.

Si bien el tema con respecto a la felicidad se encuentra abordado infinitas veces, en esta oportunidad buscamos reafirmar y ampliar la mirada frente a los diferentes modelos de felicidad que manifiestan los entrevistados. Sus respuestas permiten articular marcos teóricos que se estudiaron en la materia, a partir de los siguientes autores: Vera Poseck, con emociones positivas como optimismo; Martin Seligman y las tres vías citado en Vazquez; Tal Ben Sahar con placer y significado; y Vázquez y Hervás con el modelo de Jahoda.

46 Se aprovechó la transcripción para focalizar avances: una mayor adecuación a la situación comunicativa y al género con doble subrayado; con subrayado simple uso de un lenguaje más abstracto, menos congruente. También se focalizaron aspectos a corregir: con negrita reiteraciones, complejización innecesaria de la sintaxis. 
Con respecto a la versión inicial se observa una mayor adecuación a la consigna: desde la primera oración se sitúa al trabajo en la asignatura Psicología de la Salud y se aclara su género (el informe de una breve investigación). Se cumplen sucesivamente las fases o unidades textuales menores que caracterizan al paso Introducción: el primer párrafo logra presentar el tipo de texto y el contexto que motivó la investigación, el segundo y el tercero aclaran los pasos metodológicos; y por último se presenta (aunque sin una correcta citación) el marco teórico y conceptual.

En esta versión aparecen sintagmas que incluyen participantes abstractos (como "fortalezas, virtudes, recursos cognitivos, emocionales, vinculares y sociales", "marcos teóricos") que logran un tipo de lenguaje más cercano al académico. Estas características colaboran en ajustar los objetivos y alcances del texto, esto es, se corrió el foco puesto en los entrevistados y en la apreciación subjetiva de su grado de felicidad de la primera versión para presentar un análisis de acuerdo a cierto marco conceptual. Como ejemplo, nótese la evolución entre estos fragmentos:

Primera versión:

"(...) buscamos reafirmar y ampliar la mirada frente a las diferentes posibilidades y formas que encuentran las personas de esta categoría para ser felices"

Última versión:

"buscamos reafirmar y ampliar la mirada frente a los diferentes modelos de felicidad que manifiestan los entrevistados. Sus respuestas permiten articular marcos teóricos $(\ldots)^{\prime \prime}$

También se superaron en parte ciertas dificultades en el nivel léxico-gramatical: se corrigió el uso de los verboides, se logró una menor redundancia (aunque sigue habiendo reiteración léxica en "adultos mayores de 60 años"). Además, a pesar de que sigue habiendo una complejización de la sintaxis inadecuada a través de la acumulación de infinitivos problemáticamente conectados ("buscamos relacionar los datos empíricos a modo de lograr una adecuada comprensión de las teorías (...), y observar sus posibles límites"), los segmentos incluidos en esta segunda versión logran una sintaxis más simple y adecuada: "En el presente trabajo se dará cuenta de una breve investigación acerca de qué es la felicidad (...)", "Sus respuestas permiten articular marcos teóricos que se estudiaron en la materia (...)".

\section{Reflexiones finales}

Dado que las características de los géneros utilizados para la enseñanza y el aprendizaje en la Universidad tienen características muy diferentes de los que los estudiantes han podido abordar en otros ámbitos, la enseñanza de la lectura y la escritura a lo largo de la carrera universitaria con carácter propedéutico se vuelve imprescindible. Esta enseñanza permite que los estudiantes logren mejores resultados en el aprendizaje de los contenidos disciplinares, dado que estas prácticas les permiten trabajar mejor la construcción de conocimiento en cada materia. 
Un programa como el presentado aquí requiere de la descripción de géneros y caracterización del lenguaje específico, más una propuesta didáctica que favorezca los procesos de enseñanza y aprendizaje de este tipo de contenidos con impacto en el aprendizaje de contenidos de la materia en que se inserte.

Este tipo de trabajo demanda el compromiso de todos los docentes, incluyendo a los docentes de Letras que se asocian a la enseñanza tanto como a quienes están a cargo de la materia específica de la carrera. Asimismo, requiere compromiso de los estudiantes, pues deben asumir este trabajo como intrínseco a la formación profesional. Sin embargo, todo esto no es posible si no existen la decisión y el compromiso por parte de la institución. Son los responsables más altos los que hacen que un programa de lectura y escritura a lo largo de las carreras universitarias puedan tener lugar en el plan de formación de estudiantes, con el objetivo de prepararlos para lograr su graduación.

En efecto, como pudo observarse en el caso analizado, el conjunto de conocimientos necesarios para la realización de los textos solicitados no puede transmitirse teóricamente sino que requieren de una práctica de reconocimiento de la estructura y recursos lingüísticos característicos de un género y de la reflexión sobre las propias producciones y un ejercicio sostenido y reflexivo en el manejo de la escritura académica que, como se señaló antes, corresponde a la universidad.

A partir de lo mostrado en los casos de análisis, puede sostenerse que la metodología en la implementación del Programa fue altamente favorable: los alumnos pudieron volver sobre sus escritos y reflexionar acerca de las dificultades gracias a que se pudieron realizar actividades de deconstrucción y de edición conjunta a partir de la negociación entre pares. Cabe señalar, sin embargo, que estos avances son los primeros realizados por los estudiantes, en el primer cuatrimestre de cursado de la carrera, dando cuenta de un aprendizaje incipiente en relación con las convenciones genéricas y el tipo de lenguaje adecuado al contexto. Desde luego que no son suficientes, razón por la cual se subraya la necesidad de que la enseñanza de este tipo de discurso y sus géneros se extienda a toda la carrera.

Como ya se señaló, el trabajo interdisciplinario entre especialistas de las disciplinas en juego en la carrera y de las ciencias del lenguaje mejora considerablemente las condiciones para el aprendizaje de los géneros académicos y profesionales objeto de enseñanza en el nivel de los estudios superiores.

\section{Bibliografía}

Adelstein, A. \& Kuguel, I. Los textos académicos en el nivel universitario. Los Polvorines: Universidad Nacional de General Sarmiento, 2004.

Arnoux, E. (Dir.) Escritura y producción de conocimiento en las carreras de posgrado. Buenos Aires: Santiago Arcos, 2009.

Arnoux, E., Nogueira, S. \& Silvestri, A. "La escritura producida a partir de la lectura de textos polifónicos. Evaluación del desempeño de grupos con diferente entrenamiento lector previo", en M. C. Martínez (comp.) Aprendizaje de la argumentación razonada, Cali, Cátedra UNESCO y Universidad de Cali, 200 I. 
Arnoux, E., di Stefano, M. \& Pereira, C. La lectura y la escritura en la universidad. Buenos Aires: EUDEBA, 2002.

Arnoux, E., Nogueira, S. \& Silvestri, A. "La construcción de representaciones enunciativas: el reconocimiento de voces en la comprensión de textos polifónicos", Revista Signos 35(5I-52), 2002, págs. I29- 448.

Banks, D. The development of Scientific Writing. Linguistic Features and Historical Context. London: Equinox, 2008.

Bawarshi, A.S. \& Reiff, M.J. Genre. An Introduction to History, Theory, Research and Pedagogy. Colorado/Indiana: Parlor Press and WAC Clearinghouse, 20 I0. Disponible en http://wac.colostate.edu/books/genre/

Bazerman, Ch.; Bonini, A \& Figueredo, D. (Eds) (Genre in a changing world. Colorado/Indiana: Parlor Press and WAC Clearinghouse, 2009. Disponible en http://wac.colostate.edu/books/genre/

Bazerman, Ch.; Krut, R.; Lunsford, K; McLeod, S.; Null, S.; Rogers, P. \& Stansell, A. (Eds.) Traditions of Writing Research. New York/London: Routledge, 2010.

Braidot, N.; Moyano, E.; Natale, L. \& Roitter, S. "Enseñanza de la lectura y la escritura como política institucional a lo largo de las carreras de ingeniería del IDEI-UNGS". En: Trabajos completos, 2008, Cód. 368. VI CAEDI, UNSa-UCASAL-COPAIPA. Salta, setiembre 2008.

Carlino, P. La experiencia de escribir una tesis: contextos que la vuelven más difícil. Anales del Instituto de Lingüística, Vol. xxiv-Xxv-xxvi: 4I-62, 2005. Disponible en http://www.escrituraylectura.com.ar/posgrado/ articulos.htm

Carlino, P. Escribir, leer y aprender en la universidad. Una introducción a la afabetización académica. Buenos Aires: F.C.E., 2005.

Carlino, P. (Coord.) "Procesos y prácticas de escritura en la educación superior". Signo \& Seña N 16, 2006. Buenos Aires: Facultad de Filosofía y Letras, UBA. Disponible en: http://www.escrituraylectura.com.arl posgrado/revistas/SySI6.pdf

Coffin, C. \& Donohue, J.P. (Eds) English for Academic Purposes: contributions from Systemic Functional Linguistics and Academic Literacies. ESP Volume II, Issue I, 2012.

Cubo de Severino, L. "Evaluación de estrategias retóricas en la comprensión de manuales universitarios". $\underline{\text { RILL }}$ NoI5, 2002, págs. 69-84.

De Lelia, C. \& Ezcurra, A. M. (Dir). Competencias cognitivas de alumnos del penúltimo año de educación media. Instituto de Estudio Acción Social. Investigación destinada a la Universidad Nacional de General Sarmiento, 1994.

Di Stéfano, M. \& Pereira, M.C. "Representaciones sociales de la lectura en el proceso de comprensión lectora", Signo \& Seña Nº 8, 1997, Perspectivas en Psicolingüística: modelos, objetos y métodos. Instituto de Lingüística, UBA, págs. 318-340.

Di Stéfano, M. \& Pereira, M.C. "La enseñanza de la lectura y la escritura en el nivel superior: procesos, prácticas y representaciones sociales" en: Paula Carlino (Coord) Textos en Contexto: Leer y escribir en la universidad, Asociación Internacional de Lectura, Lectura y Vida, Buenos Aires, 2004, págs. 23-4I. 
Giudice, J. \& Moyano, E. "Apropiación del discurso de la economía: Análisis evolutivo de un caso". En: Barbara, L. \& Moyano, E. (Eds.), Textos y lenguaje académico. Exploraciones sistémico-funcionales en portugués y español. Los Polvorines/San Pablo: UNGS-PUCSP, 201 I, págs. 91 - II 2.

Ezcurra, A. (Dir). Competencias cognitivas de alumnos de primer ingreso universitario potencial (en actividades de comprensión y producción de textos de tipo expositivo y argumentativo de género académico). Los Polvorines: Universidad Nacional de General Sarmiento Investigación de la Unidad Pedagógica Universitaria, 1995.

Halliday, M.A.K. El lenguaje como semiótica social. México: FCE, 1982.

Halliday, M.A.K. "Towards a Language-based theory of learning". Linguistics and Education, 5, 1993, págs. 93- I I 6.

Halliday, M.A.K. "The construction of Knowledge and Value in the Grammar of Scientific Discourse: Charles Darwin's The Origin of the Species". En Halliday, M. \& J.R. Martin Writing science: Literacy and discursive power. Pittsburgh: University of Pittsburgh Press, 1993, págs. 86-105.

Halliday, M.A.K. An Introduction to Functional Grammar, 2nd ed. London: Arnold, 1994.

Halliday, M. "Things and relations: Regrammatizing experience as technical knowledge". In: Martin, Jim; Veel, R. (Eds.) Reading science. London: Routledge, 1998, págs. 185-236.

Halliday, M.A.K. "Computing Meanings: Some Reflections on Past Experience and Present Prospects". En: Computational and Quantitative Studies. Collected Works of M.A.K. Halliday, Vol 6, Editado por Jonathan J. Webster. London: Continuum, 2005, págs. 239-267.

Halliday, M.A.K. "The Notion of 'Context' in Language Education". En: Language in Education. Collected Works of M.A.K. Halliday, Vol 9, Editado por Jonathan J. Webster. London: Continuum, 2007, págs. 269-290.

Halliday, M.A.K. \& Hasan, R. Language, context, and text: Aspects of language in a social-semiotic perspective. Deakin University Press, 1985.

Halliday, M. \& Martin, J.R. Writing science: Literacy and discursive power. Pittsburgh: University of Pittsburgh Press, 1993.

Hood, S. Appraising research: evaluation in academic writing. London: Palgrave Macmillan, $20 \mathrm{I} 0$.

Hyland, K. Teaching and Researching Writing. London: Longman, 2002.

Hyland, K. \& Hamp Lyons, L. "EAP: issues and directions". Journal of English for Academic Purposes, I, 2002, págs. I - 12.

Hyon, S. "Genre in three traditions: implications for ESL". TESOL Quarterly 30(4), 1996, págs. 693-722.

Korner, H.; McInnes, D. \& Rose, D. Science literacy. NSW: NSW AMES, 2007.

Martin, J.R. "Analyzing genre: functional parameters". En: Christie, F. \& Martin, J.R. (ed). Genre and institutions: Social processes in the workplace and school. London: Continuum, 1997, págs. 3-39.

Martin, J.R. "Modelling context: a crooked path of progress in contextual linguistics (Sydney SFL)". En Ghadessy, M. (Ed). Text and Context in Functional Linguistics. Amsterdam: Benjamins (CILT Series IV), 1999, págs. 25-6I. 
Martin, J. R. "Mentoring semogenesis: 'Genre-based' literacy pedagogy". En Christie, F. (Ed.) Pedagogy and the shaping of consciousness. Linguistic and social processes, 1999b, págs. 123- 155. London: Continuum.

Martin, J.R. "A context for genre: modelling social processes in functional linguistics". En: Devilliers, J \& R. Stainton (Eds.) Communication in Linguistics: papers in honour of Michael Gregory. Toronto: GREF (Theoria Series 10), 200I, págs. 87-328.

Martin, J.R. "Genre and language learning: A social semiotic perspective". Linguistics and Education 20, 2009, págs. |0-21.

Martin, J.R. "Evolving systemic functional linguistics: beyond the clause". Functional Linguistics, 1:3, 2014.

Martin, J.R. \& Rose, D. Genre Relations: mapping culture. London, Equinox, 2008.

Martin, J.R. \& Veel, R. (Eds.) Reading science. London: Routledge, 1998.

Martin, J.R. \& White, P.R.R. The language of evaluation. Appraisal in English. London, Palgrave, 2005.

Molina Natera, $\vee$. "Centros de escritura: una mirada retrospectiva para entender el presente y futuro de estos programas en el contexto latinoamericano". Revista Legenda Vol 18 NI 8, 20 I4, págs. 9-33.

Moyano, E.I. Evaluación Diagnóstica Sumativa del Taller de Lecto-escritura del curso de aprestamiento Universitario de la UNGS, Informe Final de Resultados. Los Polvorines: Publicación interna Secretaría Académica, IDH-UNGS, 2003.

Moyano, E.I. "La escritura académica: una tarea interdisciplinaria a lo largo del curriculum universitario". Texturas 4 (4), 2004, págs. 109-120.

Moyano, E.l. "Enseñanza de habilidades discursivas en español en contexto pre-universitario: Una aproximación desde la LSF". Revista Signos 40 (65), 2007, págs. 573- 608.

Moyano, E.I. "Negotiating Genre: Lecturer's Awareness in Genre Across the Curriculum Project at the University Level". En: Bazerman, Charles, Bonini, Adair, and Figueiredo, Débora. 2009. Genre in a Changing World. Perspectives on Writing, 2009, pp. 449-464. Fort Collins, Colorado: The WAC Clearinghouse and Parlor Press. Disponible en: http://wac.colostate.edu/books/genre/

Moyano, E.I. "Escritura académica a lo largo de la carrera: un programa institucional. Revista Signos 43 (74), 20 I0, págs.465-488.

Moyano, E.l. "Deconstrucción y Edición Conjuntas en la enseñanza de la escritura. La reflexión sobre género y discurso en la formación académica y profesional'. En: Anais VI Simpósio Internacional de Estudos de Ganeros Textuais (SIGET), 20I I. Disponible en: http://www.cchla.ufrn.br/visiget/

Moyano, E.I. "Argumentación en Economía: Negociación de una Interpretación”. Revista R.I.L.L. N 17, vol I y 2, 2012. Disponible en: http://www.insil.com.ar/rill2012.asp

Moyano, E.I. "Hacia la caracterización de géneros profesionales: algunas reflexiones teórico-metodológicas". En: Nothstein, S.; Pereira, M.C. \& Valente, E. (Comps) Libro de Actas del Congreso Regional de la Cátedra UNESCO en Lectura y Escritura: "Cultura Escrita y Políticas Pedagógicas en las Sociedades Latinoamericanas Actuales". 
Los Polvorines: Universidad Nacional de General Sarmiento, 20 I2, págs. I567-I586. Disponible en: http:// www.ungs.edu.ar/ms idh/wp-content/uploads/201 I/I I/Libro-de-Actas2.pdf

Moyano, E.l. "Proyección congruente y metafórica en las discusiones de artículos científicos de tres disciplinas en español'. En: Vian Jr., O. \& Caltabiano, C. (Orgs.) Língua(gem) e suas múltiplas faces. San Pablo: Mercado de Letras, 2013, págs. 109-133.

Moyano, E.l. "La Discusión en artículos de Microbiología: género, compromiso y construcción del conocimiento". Revista Onomázein, Número Especial IX ALSFAL, 20 I4, págs. 16 I - 185. Disponible en: http://www.onomazein. net/03 Numeros/Nespecial IXALSFAL/Nespecial IXalsfal.html

Moyano, E.I. "Patrones de realización de la proyección en la Discusión de artículos de investigación producidos en español en dos disciplinas". Revista D.E.L.T.A. 3I (I), 20I5, págs. |43- |83. Disponible en:

http://www.scielo.br/scielo.php?script=sci arttext\&pid=S0 102-445020 I $5000100143 \&$ Ing=en\&tlng=es .

Moyano, E.I. \& Natale, L. "Teaching Academic Literacy across the University Curriculum as Institutional Policy. The case of the Universidad Nacional de General Sarmiento (Argentina)". En: Thaiss, Ch, G. Bräuer; P. Carlino; L. Ganobcsik-Williams, and A. Sinha. Writing Programs Worldwide: Profiles of Academic Writing in Many Places. Perspectives on Writing. Fort Collins, Colorado: The WAC Clearinghouse and Parlor Press, 20 I2. Disponible en http://wac.colostate.edu/books/wrab20I I/

Navarro, F. "Trayectorias de formación en lectura y escritura disciplinar en carreras universitarias de humanidades: diagnóstico y propuesta institucional”. Revista Mexicana de Investigación Educativa 18 (58), 20I3, págs. 709-734.

Núñez Cortés, J.A. "Una aproximación a los centros de escritura en lberoamérica". Revista Legenda, Vol. 17 № 17 , 2013, págs. 64-102.

Oteiza, T. "Solidaridad ideológica en el discurso de la historia: Tensión entre orientaciones monoglósicas y heteroglósicas". Revista Signos 42 (70), 2009, págs. 219-244.

Oteiza, T. "Patrones valorativos en el discurso oficial de Derechos Humanos en Chile: dando valor al pasado y construyendo memorias históricas en la sociedad". Discurso y Sociedad 4 (I), 20 I0, págs. I5 I - 83.

Padilla, C. "Argumentación académica: La escritura de ponencias en el marco de una asignatura universitaria", Actas del XI Congreso Nacional de Lingüística, SAL, Santa Fe, Arg.: Univ. Nac. del Litoral, 2009.

Pereira, C. \& di Stefano, M. "El taller de escritura en posgrado: Representaciones sociales e interacción entre pares". Rev. Signos v.40 (64), 2007, págs. 405-430.

Parodi, G. Alfabetización académica y profesional en el Siglo XXI: Leer y escribir desde las disciplinas. Santiago de Chile: Ariel, 2010.

Rose, D. \& Martin, J.R. Learning to Write, Reading to Learn. Genre, Knowledge and Pedagogy in the Sydney School. UK: Equinox, 2012.

Swales, J. Genre analysis. English in academic and research settings. Cambridge: Cambridge University Press, 1990. 
Swales, J. "Occluded Genres in the Academy: The Case of the Submission Letter". En: Ventola, E. \& Mauranen, A. (Eds.) Academic Writing. Intercultural and Textual Issues. Amsterdam: Benjamins, 1996, págs. 45-58.

Thaiss, Ch. "The international WACMID mapping project: objectives, methods, and early results". En Bazerman, Ch.; Krut, R.; Lunsford, K.; McLeod, S.; Null, S.; Rogers, P \& Stansell, A. Traditions of Writing Research. New York / London: Routledge, 2010 , págs. 252-264.

Thaiss, Ch.; G. Bräuer; P. Carlino; Ganobcsik-Williams, L. \& Sinha, A. Writing Programs Worldwide: Profiles of Academic Writing in Many Places. Perspectives on Writing. Fort Collins, Colorado: The WAC Clearinghouse and Parlor Press, 20 I2. Disponible en http://wac.colostate.edu/books/wrab20I I/

UNLu. La Lectura y Escritura como prácticas académicas universitarias, Luján, Bs. As: UNLU, Departamento de Educación, 200 I. Disponible en: http://www.unlu.edu.ar/ redecom/borrador.htm

Uslenghi de Murga; Padilla, C.; Ameijide, M.D. \& Douglas, S. "Discurso estudiantil: Representaciones acerca de las competencias discursivas. Análisis crítico del discurso estudiantil en el nivel universitario. Oralidad, lectura y escritura: categorización de dificultades." RILL N 15 , 2002, págs. 85- 103.

Vázquez, A.; Jakob, l.; Novo, M.C. \& Pelizza, L. (Comp.) Lectura, escritura y aprendizaje disciplinar. Río IV: UniRío Editora, Universidad Nacional de Río Cuarto, 2012. Disponible en: http://www.unrc.edu.arlunrc/digtal/libro jornadas unesco unrc 2010.pdf

Vigotsky, L. Pensamiento y lenguaje. Buenos Aires: Fausto, 1998.

Wertsch, J. Voces de la Mente. Un Enfoque Sociocultural para el Estudio de la Acción Mediada. Madrid: Visor, I99I.

Wignell, P. On the discourse of social science. Darwin: Charles Darwin University Press, 2007.

Zalba, E.M. "La palabra interdicta: problemas de producción discursiva en estudiantes universitarios". Ponencia presentada en el Simposio Internacional Lectura y escritura: nuevos desafios, Facultad de Ciencias Políticas y Sociales \& Facultad de Filosofía y Letras de la Universidad Nacional de Cuyo. Mendoza, mayo de 2002. 